\title{
Subspace Condition for Bernstein's Lethargy Theorem
}

\author{
Asuman Güven AKSOY \\ corresponding author, Claremont McKenna College \\ Department of Mathematics, Claremont, CA 91711, USA \\ E-mail: aaksoy@cmc.edu \\ Monairah AL-ANSARI \\ Claremont Graduate University \\ Institute of Mathematical Sciences, Claremont, CA 91711, USA \\ E-mail: monairah.alansari@cgu.edu \\ Caleb CASE \\ Claremont McKenna College \\ Department of Mathematics, Claremont, CA 91711, USA \\ E-mail: caleb.case16@cmc.edu \\ Qidi PENG \\ Claremont Graduate University \\ Institute of Mathematical Sciences, Claremont, CA 91711, USA \\ E-mail: qidi.peng@cgu.edu
}

\begin{abstract}
In this paper, we consider a condition on subspaces in order to improve bounds given in the Bernstein's Lethargy Theorem (BLT) for Banach spaces. Let $d_{1} \geq d_{2} \geq \ldots d_{n} \geq \cdots>0$ be an infinite sequence of numbers converging to 0 , and let $Y_{1} \subset Y_{2} \subset \cdots \subset Y_{n} \subset \cdots \subset X$ be a sequence of closed nested subspaces in a Banach space $X$ with the property that $\bar{Y}_{n} \subset Y_{n+1}$ for all $n \geq 1$. We prove that for any $c \in(0,1]$, there exists an element $x_{c} \in X$ such that

$$
c d_{n} \leq \rho\left(x_{c}, Y_{n}\right) \leq \min (4, \tilde{a}) c d_{n} .
$$

Here, $\rho\left(x, Y_{n}\right)=\inf \left\{\|x-y\|: y \in Y_{n}\right\}$,

$$
\tilde{a}=\sup _{i \geq 1} \sup _{\left\{q_{i}\right\}}\left\{a_{n_{i+1}-1}^{-3}\right\}
$$
\end{abstract}


where the sequence $\left\{a_{n}\right\}$ is defined as: for all $n \geq 1$,

$$
a_{n}=\inf _{l \geq n} \inf _{q \in\left\langle q_{l}, q_{l+1}, \ldots\right\rangle} \frac{\rho\left(q, Y_{l}\right)}{\|q\|}
$$

in which each point $q_{n}$ is taken from $Y_{n+1} \backslash Y_{n}$, and satisfies $\inf _{n \geq 1} a_{n}>0$. The sequence $\left\{n_{i}\right\}_{i \geq 1}$ is given by

$$
n_{1}=1 ; n_{i+1}=\min \left\{n \geq 1: \frac{d_{n}}{a_{n}^{2}} \leq d_{n_{i}}\right\}, i \geq 1 .
$$

『

\section{Introduction}

Bernstein's Lethargy Theorem [7] involves finding approximations of an element in a space $X$ when those approximations are limited to some sequence of subspaces. Before we can compare approximations, we need a function to determine how close an approximation is to the desired target. In the following we define a distance function which we call the $\rho$-function:

Definition 1 Let $(X,\|\cdot\|)$ be a Banach space, and let $S$ be a subspace of $X$. Then, for any point $x \in X$, we can define the distance from $x$ to $S$ as

$$
\operatorname{dist}(x, S)=\rho(x, S)=\inf _{y \in S}\|x-y\| .
$$

If $Y_{1} \subset Y_{2} \subset \ldots$ is a sequence of strictly embedded linear subspaces of $X$, then for each $x \in X$, there exists a non-increasing sequence of best approximation errors

$$
\rho\left(x, Y_{1}\right) \geq \rho\left(x, Y_{2}\right) \geq \ldots
$$

The general objective is to characterize these sequences of best approximation errors. For example, one can ask if it is true that for any non-increasing sequence $\left\{d_{n}\right\}$ with $\lim _{n \rightarrow \infty} d_{n}=0$, there exists an element $x \in X$ such that

$$
\rho\left(x, Y_{n}\right)=d_{n} \quad \text { for all } n=1,2, \ldots
$$

Bernstein 7] proved that in the case $X=C[a, b]$ and $Y_{n}=P_{n}$, the space of polynomials of degree at most $n$, any sequence converging to zero is a sequence of best approximations. This theorem is sometimes referred to as Bernstein's Lethargy Theorem (BLT) and it has been applied to the theory of quasi analytic functions in several complex variables [15] and used in the constructive theory of functions [19]. Note that the density of polynomials in $C[0,1]$ (Weierstrass Approximation Theorem [10]) implies that $\lim _{n \rightarrow \infty} \rho\left(f, P_{n}\right)=0$. However, Weierstrass

Mathematics Subject Classification (2000): 41A25, 41A50, 41A65.

Key words: Best Approximation, Bernstein's Lethargy Theorem, Banach Spaces. 
Approximation Theorem gives no information about the speed of convergence for $\rho\left(f, Y_{n}\right)$. Following the proof of Bernstein, Timan [20] extended his result to an arbitrary system of strictly embedded finite-dimensional subspaces $Y_{n}$. Later Shapiro [18, replacing $C[0,1]$ with an arbitrary infinite-dimensional Banach space $(X,\|\cdot\|)$ and the sequence of $n$-dimensional subspaces of polynomials of degree $\leq n$ by a sequence $\left\{Y_{n}\right\}$ where $Y_{1} \subset Y_{2} \subset \cdots$ are strictly embedded closed subspaces of $X$, showed that in this setting, for each null sequence $\left\{d_{n}\right\}$ of non-negative numbers, there is a vector $x \in X$ such that

$$
\rho\left(x, Y_{n}\right) \neq O\left(d_{n}\right), \text { as } n \rightarrow \infty .
$$

Thus, there is no $M>0$ such that $\rho\left(x, Y_{n}\right) \leq M d_{n}$ for all $n$. In other words, $\rho\left(x, Y_{n}\right)$ can decay arbitrarily slowly. This result was strengthened by Tyuriemskih 22] who established that the sequence of best approximations may converge to zero at an arbitrary slow rate: for any expanding sequence $\left\{Y_{n}\right\}$ of subspaces and for any sequence $\left\{d_{n}\right\}$ of positive numbers converging to zero, he constructed an element $x \in X$ such that $\lim _{n \rightarrow \infty} \rho\left(x, Y_{n}\right)=0$ and $\rho\left(x, Y_{n}\right) \geq d_{n}$ for all $n$. For a generalization of Shapiro's Theorem we refer the reader to [6]. For an application of Tyuriemskih's Theorem to convergence of sequence of bounded linear operators, consult [11]. For other versions of Bernstein's Lethargy Theorem, see [1, 3, 2, 5, 14, 16.

We now consider the following well-known Bernstein's Lethargy Theorem [7, stated for the case of finite-dimensional subspaces of a Banach space $X$.

Theorem 2 (Lethargy) Given a Banach space $X$ and a series of nested finitedimensional subspaces $Y_{1} \subset Y_{2} \subset \cdots \subset X$. If $\left\{d_{k}\right\}_{k \geq 1}$ is a monotone decreasing sequence converging to 0 , then there exists a point $x \in X$ such that $\rho\left(x, Y_{k}\right)=d_{k}$ for all $k \geq 1$.

The above theorem can be extended to infinite-dimensional subspaces, by considering some extra conditions. Borodin [8] has provided two sets of conditions. One condition is on the sequence $\left\{d_{n}\right\}$ and the other on both the subspaces $\left\{Y_{n}\right\}$ and the sequence $\left\{d_{n}\right\}$. In both cases, he proves the existence of an element $x \in X$ with $\rho\left(x, Y_{k}\right)=d_{k}, k \geq 1$. These two results are explicitly presented as follows.

Theorem 3 (see [8]) Let $X$ be an arbitrary infinite-dimensional Banach space, $Y_{1} \subset Y_{2} \subset \ldots$ be an arbitrary system of strictly embedded subspaces in $X$, and the number sequence $\left\{d_{n}\right\}$ be such that

$$
d_{n}>\sum_{k=n+1}^{\infty} d_{k}
$$

for every positive integer $n \geq n_{0}$ for which $d_{n}>0$. Then there exists an element $x \in X$ such that $\rho\left(x, Y_{n}\right)=d_{n}$ for $n \geq 1$. 
Theorem 4 (see [8]) Let $d_{0} \geq d_{1} \geq d_{2} \geq \cdots>0$ be a non-increasing sequence converging to 0 and $Y_{1} \subset Y_{2} \subset \cdots \subset X$ be a system of strictly nested subspaces of an infinite-dimensional Banach space $X$ that meets the following property: there exists a series of nonzero elements $q_{n}$ such that $q_{n} \in Y_{n+1} \backslash Y_{n}$, and the following inequality

$$
\|q\| \leq \frac{d_{k-1}}{d_{k}} \rho\left(q, Y_{k}\right)
$$

holds for all $k \in \mathbb{N}$ and any nonzero element $q$ in the linear span $\left\langle q_{k}, q_{k+1}, \ldots\right\rangle$. Then there is some element $x$ in the closed linear span $\overline{\left\langle q_{1}, q_{2}, \ldots\right\rangle}$ satisfying

$$
\rho\left(x, Y_{n}\right)=d_{n} \text { for all } n \geq 1 .
$$

Recently Konyagin [12 showed that under the same assumptions in Theorem 3. except that the sequence $\left\{d_{n}\right\}$ can go to 0 with arbitrary rate, there is $x \in X$ such that

$$
d_{n} \leq \rho\left(x, Y_{n}\right) \leq 8 d_{n}, \text { for } n \geq 1 .
$$

The proof is based on Theorem 3. Note that the statements in Theorem 4 are similar to that of Theorem 3. We can now adapt the idea of the proof of Konyagin's [12] with the Borodin's theorem [8] to improve the bounds of $\rho\left(x, Y_{n}\right)$ in (3).

In Konyagin's paper [12, it is assumed that $Y_{n}$ are closed and strictly increasing. In Borodin's paper, this is not specified, but from the proof of his theorem it is clear that his proof works only under the assumption that $\overline{Y_{n}}$ is strictly included in $Y_{n+1}$. Necessity of this assumption on subspaces is illustrated by the following:

Example 5 Let $X=L^{\infty}[0,1]$ and consider $C[0,1] \subset L^{\infty}[0,1]$. Define the subspaces of $X$ as follows:

- $Y_{1}=$ all polynomials

- $Y_{2}=\operatorname{span}\left[Y_{1} \cup f_{1}\right]$, where $f_{1} \in C[0,1] \backslash Y_{1}$,

- $Y_{n+1}=\operatorname{span}\left[Y_{n} \cup f_{n}\right]$ where $f_{n} \in C[0,1] \backslash Y_{n}$.

Observe that by Weierstrass Theorem, $\overline{Y_{n}}=C[0,1]$ for any $n \geq 1$. Take any $f \in L^{\infty}[0,1]$ and consider the following cases:

1. If $f \in C[0,1]$, then

$$
\operatorname{dist}\left(f, Y_{n}\right)=\operatorname{dist}(f, C[0,1])=0 \text { for any } n \geq 1 .
$$

2. If $f \in L^{\infty}[0,1] \backslash C[0,1]$, then

$$
\operatorname{dist}\left(f, Y_{n}\right)=\operatorname{dist}(f, C[0,1])=d>0 \quad(\text { independent of } n) .
$$

Hence in this case BLT does not hold. (Note that in the above, we used the fact that $\operatorname{dist}(f, Y)=\operatorname{dist}(f, \bar{Y})$.) 
Note that Borodin's condition on sequence $\left\{d_{n}\right\}$, namely $d_{n}>\sum_{k=n+1}^{\infty} d_{k}$ is not satisfied when $d_{n}=\frac{1}{2^{n}}$, however it is satisfied when $d_{n}=\frac{1}{(2+\epsilon)^{n}}$ for $\epsilon>0$. Thus, it is natural to ask whether the condition (11) is necessary for the results in Theorem 3 to hold?

In 4], it is shown that by weakening the condition (1) in Theorem 3 above yields to an improvement of the bounds in the inequality (3) in Konyagin's theorem. In this paper, we take a different approach. We concentrate on the Borodin's second condition on subspaces, namely on the inequality (2) of Theorem 4 above and obtain better bounds for the inequalities in (3). The statements in Theorem 4 are similar to that of Theorem 3. thus, we can now adapt the idea of the proof of Konyagin's [12] with the Borodin's theorem [8] to improve the bounds of $\rho\left(x, Y_{n}\right)$ in (3).

\section{Main Result}

Let $X$ be an arbitrary infinite-dimensional Banach space. Given $Y_{1} \subset Y_{2} \subset$ $\cdots \subset X$, an arbitrary system of strictly embedded closed subspaces and $d_{1} \geq$ $d_{2} \geq \cdots \geq 0$, a non-increasing sequence converging to 0 . The goal of this paper is to improve Konyagin's result (3) under conditions on subspaces $\left\{Y_{n}\right\}$ and $\left\{d_{n}\right\}$. It is worth noting that if $\left\{Y_{n}\right\}$ and $\left\{d_{n}\right\}$ are finite sequences, we have the best approximations of the sequence $d_{n}$ in terms of the distances $\rho\left(x, Y_{n}\right)$, i.e., the following lemma holds:

Lemma 6 Let $d_{1}>d_{2}>\cdots>d_{n}>0$ be a finite decreasing sequence and $Y_{1} \subset Y_{2} \subset \cdots \subset Y_{n}$ be a system of strictly nested closed subspaces of Banach space $X$. Then for any $c \in(0,1]$, there exists an element $x_{c} \in X$ such that $\rho\left(x_{c}, Y_{k}\right)=c d_{k}$, for $k=1, \ldots n$.

Proof. First, from [8] and [20], we see Lemma 6] is true for $c=1$. Next for any $c \in(0,1]$, let $\tilde{d}_{k}=c d_{k}$. It is easy to see the sequence of numbers $\left\{\tilde{d}_{k}\right\}$ satisfies Lemma 6, therefore there exists an element $x_{c} \in X$ such that $\rho\left(x, Y_{k}\right)=\tilde{d}_{k}$, for $k=1, \ldots, n$.

Now we consider the case when $\left\{Y_{n}\right\}$ and $\left\{d_{n}\right\}$ are infinite sequences and state our main result.

Theorem 7 Let $X$ be an arbitrary infinite-dimensional Banach space, and let $Y_{1} \subset Y_{2} \subset \cdots \subset X$ be an arbitrary system of strictly embedded closed linear subspaces. Let $\left\{d_{n}\right\}$ be a non-increasing sequence of real numbers converging to 0. Assume that, for any sequence of elements $q_{i}$ such that $q_{n} \in Y_{n+1} \backslash Y_{n}$ for all $n$, we have that

$$
\inf _{n \geq 1} a_{n}>0
$$


where for each $n \geq 1, a_{n}$ is defined by

$$
a_{n}=\inf _{l \geq n} \inf _{q \in\left\langle q_{l}, q_{l+1}, \ldots\right\rangle} \frac{\rho\left(q, Y_{l}\right)}{\|q\|}
$$

for these elements $q_{i}$. Then for any constant $c \in(0,1]$, there exists an element $x_{c} \in X$ such that

$$
c d_{n} \leq \rho\left(x_{c}, Y_{n}\right) \leq \min (4, \tilde{a}) c d_{n},
$$

where

$$
\tilde{a}=\sup _{i \geq 1} \sup _{\left\{q_{i}\right\}}\left\{a_{n_{i+1}-1}^{-3}\right\}
$$

and $n_{i}$ satisfies

$$
\begin{aligned}
& n_{1}=1 \\
& n_{i+1}=\min \left\{n \geq 1: \frac{d_{n}}{a_{n}^{2}} \leq d_{n_{i}}\right\}, i \geq 1 .
\end{aligned}
$$

Proof. If $d_{n}=0$ for some $n$, then Theorem 7 holds by using Lemma 1 in 8 and Lemma 6. Thus, we will assume that $d_{n}>0$ for all $n \geq 1$. Take the sequence $\left\{n_{i}\right\}$ defined in (6). Define a sequence of positive integers $\left\{j_{i}\right\}$ such that

$$
j_{1}=1, j_{i+1}= \begin{cases}j_{i}+1 & \text { if } n_{i+1}=n_{i} ; \quad \text { for } i \geq 1 \\ j_{i}+2 & \text { if } n_{i+1}>n_{i},\end{cases}
$$

Let

$$
m_{j}= \begin{cases}n_{i} & \text { if } j=j_{i} \\ n_{i+1}-1 & \text { if } j_{i}<j<j_{i+1} .\end{cases}
$$

Clearly the sequence $\left\{m_{j}\right\}_{j \geq 1}$ is strictly increasing. Now, we define the sequences of subspaces $\left\{Z_{j}\right\}_{j \geq 1}$ and numbers $\left\{e_{j}\right\}_{j \geq 1}$ to be

$$
\begin{aligned}
& Z_{j}=Y_{m_{j}}, \\
& e_{j}= \begin{cases}\frac{c}{a_{m_{j+1}}} d_{n_{i}} & \text { if } j=j_{i} \text { for some } i ; \\
c d_{n_{i}} & \text { if } j_{i}<j<j_{i+1} .\end{cases}
\end{aligned}
$$

Hence, for any $j \geq 1,3$ cases follow:

Case 1:

if $j_{i}<j<j_{i+1}$ for some $i$, then $j+1=j_{i+1}$. By the definition of $e_{j}$, the facts that $n_{i+1}=m_{j+1}$ and $\left\{a_{n}\right\}_{n}$ is increasing, we obtain

$$
e_{j+1}=\frac{c}{a_{m_{j+2}}} d_{n_{i+1}} \leq \frac{c}{a_{m_{j+2}}} a_{n_{i+1}}^{2} d_{n_{i}}=\frac{c}{a_{m_{j+2}}} a_{m_{j+1}}^{2} d_{n_{i}} \leq a_{m_{j+1}} e_{j} .
$$

Case 2:

if $j=j_{i}$ for some $i$ and $j+1<j_{i+1}$, then

$$
e_{j+1}=c d_{n_{i}}=a_{m_{j+1}} e_{j} .
$$


Case 3:

if $j=j_{i}$ for some $i$ and $j+1=j_{i+1}$, then

$$
e_{j+1}=\frac{c}{a_{m_{j+2}}} d_{n_{i+1}} \leq \frac{c}{a_{m_{j+2}}} a_{n_{i+1}}^{2} d_{n_{i}}=\frac{a_{m_{j+1}} a_{n_{i+1}}^{2}}{a_{m_{j+2}}} e_{j} \leq a_{m_{j+1}} e_{j} .
$$

Thus, we conclude that

$$
e_{j+1} \leq a_{m_{j+1}} e_{j}, \text { for all } j \geq 1 .
$$

Note that for all $q \in\left\langle q_{m_{j+1}}, q_{m_{j+1}+1}, \ldots\right\rangle$,

$$
\frac{e_{j+1}}{e_{j}} \leq a_{m_{j+1}} \leq \frac{\rho\left(q, Y_{m_{j+1}}\right)}{\|q\|}=\frac{\rho\left(q, Z_{j+1}\right)}{\|q\|} .
$$

Therefore we can apply Theorem 4 to the sequence $\left\{Z_{j}\right\}_{j \geq 1}$ of subspaces and the sequence of numbers $\left\{e_{j}\right\}_{j \geq 1}$, to obtain the existence of an element $x_{c}^{\prime} \in$ $<q_{1}, q_{2}, \ldots>$ such that

$$
\rho\left(x_{c}^{\prime}, Z_{j}\right)=e_{j}, \text { for } j \geq 1 .
$$

If $n=n_{i}$ for some $i$, then for $j=j_{i}$ we have $n=m_{j}, Y_{n}=Z_{j}$ and

$$
\rho\left(x_{c}^{\prime}, Y_{n}\right)=\rho\left(x_{c}^{\prime}, Z_{j}\right)=e_{j}=\frac{c}{a_{m_{j+1}}} d_{n} .
$$

Now, let $n_{i}<n<n_{i+1}$ for some $i$ and $j=j_{i}$. Then

$$
m_{j}=n_{i}<n \leq n_{i+1}-1=m_{j+1} .
$$

It leads to the lower bound of $\rho\left(x_{c}^{\prime}, Y_{n}\right)$ in terms of $d_{n}$ :

$$
\rho\left(x_{c}^{\prime}, Y_{n}\right) \geq \rho\left(x_{c}^{\prime}, Z_{j+1}\right)=e_{j+1}=c d_{n_{i}} \geq c d_{n} .
$$

To obtain the upper bound of $\rho\left(x_{c}^{\prime}, Y_{n}\right)$ we observe from (7) that

$$
\rho\left(x_{c}^{\prime}, Y_{n}\right) \leq \rho\left(x_{c}^{\prime}, Y_{n_{i}}\right)=\rho\left(x_{c}^{\prime}, Z_{j}\right)=e_{j}=\frac{c}{a_{m_{j+1}}} d_{n_{i}} .
$$

Since $n_{i}<n_{i+1}-1<n_{i+1}$, then we have

$$
a_{n_{i+1}-1}^{2} d_{n_{i}} \leq d_{n_{i+1}-1} \leq d_{n} .
$$

Consequently,

$$
\rho\left(x_{c}^{\prime}, Y_{n}\right) \leq \frac{c}{a_{m_{j+1}}} d_{n_{i}} \leq \frac{c}{a_{n_{i+1}-1}^{2} a_{m_{j+1}}} d_{n} \leq \frac{c}{a_{n_{i+1}-1}^{3}} d_{n} .
$$

It follows from (8) and (9) that

$$
c d_{n} \leq c d_{n_{i}} \leq \rho\left(x_{c}^{\prime}, Y_{n}\right) \leq \frac{c}{a_{n_{i+1}-1}^{3}} d_{n} .
$$


Notice that $a_{n_{i+1}-1}$ only depends on the sequences $\left\{n_{i}\right\}$ and $\left\{q_{i}\right\}$. Therefore by taking supremum over $\left\{q_{i}\right\}$ and $\left\{n_{i}\right\}$ we proved

$$
c d_{n} \leq \rho\left(x_{c}^{\prime}, Y_{n}\right) \leq \tilde{a} c d_{n} .
$$

Also note that in [4] it is shown that, for the same sequences $\left\{d_{n}\right\}$ and $\left\{Y_{n}\right\}$ as in Theorem 7 there is another element $x_{c}^{\prime \prime} \in X$ such that

$$
c d_{n} \leq \rho\left(x_{c}^{\prime \prime}, Y_{n}\right) \leq 4 c d_{n} .
$$

Therefore if $\tilde{a} \leq 4$,

$$
c d_{n} \leq \rho\left(x_{c}^{\prime}, Y_{n}\right) \leq \tilde{a} c d_{n}=\min (4, \tilde{a}) c d_{n} ;
$$

if $\tilde{a}>4$,

$$
c d_{n} \leq \rho\left(x_{c}^{\prime \prime}, Y_{n}\right) \leq 4 c d_{n}=\min (4, \tilde{a}) c d_{n}
$$

Thus by taking

$$
x_{c}= \begin{cases}x_{c}^{\prime} & \text { if } \tilde{a} \leq 4 \\ x_{c}^{\prime \prime} & \text { if } \tilde{a}>4,\end{cases}
$$

we have proven Theorem 7 .

Remark 8 One can observe that the inequalities in (5) is stronger than the inequalities given by Konyagin in [12].

\section{References}

[1] Aksoy A. G, Almira J., On Shapiro's lethargy theorem and some applications. Jaen J Approx, 2014; 6(1): 87-116.

[2] Aksoy A. G, Lewicki G. , Bernstein lethargy theorem in Fréchet spaces. J. Approx. Theory 2016; 209, 58-77.

[3] Aksoy A. G, Lewicki G., Diagonal operators, s-numbers and Bernstein pairs. Note Mat 1999; 17: 209-216.

[4] Aksoy A. G., Peng Q., On a theorem of S. N. Bernstein for Banach spaces, ArXiv: 1605.04592.

[5] Almira J. M, del Toro N., Some remarks on negative results in approximation theory. In: Proceedings of the Fourth International Conference on Functional Analysis and Approximation Theory, Vol. I; 2000; Potenza; Rend. Circ. Mat. Palermo (2) Suppl., 2002, no. 68, part I, pp. 245-256.

[6] Almira J. M, Oikhberg T., Approximation schemes satisfying Shapiro's theorem. J Approx. Theory 2012; 534-571.

[7] Bernstein S. N., On the inverse problem in the theory of best approximation of continuous functions. Collected works (in Russian), Izd Akad Nauk, USSR 1954; vol II: 292-294. 
[8] Borodin P. A., On the existence of an element with given deviations from an expanding system of subspaces. Math Notes 2006; 80 (5): 621-630 (Translated from Mathematicheskie Zametki).

[9] Carleman T., Sur un théorème de Weierstrass. Arkiv Mat Astron Fys 1927; 20: 1-5.

[10] Cheney E. W., Introduction to Approximation Theory. AMS Chelsea Publishing, Amer Mathematical Society, 1966.

[11] Deutsch F, Hundal H., A generalization of Tyuriemskih's lethargy theorem and some applications. Numer Func Anal Opt 2013; 34(9): 1033-1040.

[12] Konyagin S. V., Deviation of elements of a Banach space from a system of subspaces. In: Proceedings of the Steklov Institute of Mathematics; 2014; 284 (1), pp 204-207.

[13] Lang S., Math Talks for Undergraduates. Springer, 1999.

[14] Lewicki G., A theorem of Bernstein's type for linear projections. Univ Iagellon Acta Math 1988; 27: 23-27.

[15] Pleśniak W., Quasianalytic functions in the sense of Bernstein. Dissert Math 1977; 147: 1-70.

[16] Plichko A., Rate of decay of the Bernstein numbers. Zh Mat Fiz Anal Geom 2013; 9 (1): 59-72.

[17] Rivlin T. J., An Introduction to the Approximation of Functions. Waltham, MA: Blaisdell Pub, 1969.

[18] Shapiro H. S., Some negative theorems of approximation theory. Michigan Math J 1964; 11: 211-217.

[19] Singer I., Best Approximation in Normed Linear Spaces by Elements of Linear Subspaces. Berlin. Springer-Verlag, 1970.

[20] Timan A. F., The Theory of Approximating Functions of Real Variables. Oxford. Macmillan, 1994.

[21] Tyuriemskih I. S., The B-property of Hilbert spaces. Uch Zap Kalinin Gos Pedagog Inst 1964; 39: 53-64.

[22] Tyuriemskih I. S., On one problem of S. N. Bernstein. Scientific Proceedings of Kaliningrad State Pedagog. Inst.; 1967; 52, 123-129. 